\title{
Adherencia al tratamiento de quimioterapia y radioterapia en pacientes con cáncer gástrico y colorrectal en las unidades oncológicas
}

\author{
Adherence to chemotherapy and radiotherapy treatment in \\ patients with gastric and colorectal cancer at oncology unit
}

\author{
Uldrey Hernando Cruz', Diana María Dueñas' , Karen Yulieth Martínez', Yina Paola Polo', \\ Lina Andrea Sanchez', Alix Yaneth Perdomo Romero ${ }^{2}$
}

\section{Resumen}

Objetivo: Describir los factores relacionados con la adherencia al tratamiento de quimioterapia y radioterapia en pacientes con cáncer gástrico y colorrectal. Material y Métodos: Estudio descriptivo, enfoque cuantitativo de corte transversal, con una muestra de 35 pacientes, no probabilística por conveniencia. Realizado entre junio octubre 2013, en usuarios de los Centros Oncológicos del Departamento del Huila. Para el análisis de datos se utilizó el paquete estadístico SPSS versión 19.0 a través de estadística descriptiva. Resultados: La mayoría de los encuestados supera los 51 años, poseen pareja estable y presentan asistencia y continuidad al tratamiento. Más de la mitad cuenta con apoyo económico familiar. Los pacientes tienen una adherencia favorable porque el equipo de salud demuestra actitud positiva al brindar tratamiento, manifiesta interés para lograr la asistencia de los pacientes y el personal de medicina/enfermería da recomendaciones claras, oportunas, respondiendo inquietudes derivadas del tratamiento. El $86 \%$ de la población aun cuando se siente mal, asiste puntualmente a las sesiones del tratamiento y un porcentaje similar nunca pensó abandonarlo. Conclusiones: Los factores que influyen en la adherencia al tratamiento son: pareja estable, apoyo económico familiar, percibir que el tratamiento ayuda a su recuperación, sentir motivación y respecto al equipo de salud el tener actitud positiva, demostrar interés por su asistencia y brindar recomendaciones.

Palabras clave: Cumplimiento de la medicación, Quimioradioterapia, Neoplasias gástricas, Neoplasias colorrectales, Radioterapia

\section{Abstract}

Objective: to describe factors related to treatment adherence of chemotherapy and radiotherapy in patients suffering from gastric and colorectal cancer. Materials and methods: it is a descriptive, quantitative, cross-sectional study. It was chosen a convenience non-probabilistic sample of 35 patients. The study was carried out between June and October, 2013 on patients attending oncologic centers in Huila. Data analysis was done by using SPSS version 19.0 through descriptive statistics. Results: most survey respondents are over 51, have stable partner and attend to and have treatment continuity. More than a half of them have family economic support. Patients have a favorable adherence because the health teamwork shows a positive attitude when giving treatment, show interest in making patients attend to and medicine and nursing personnel make clear, timely recommendations, replying to questions about treatment. Despite $86 \%$ of population is not feeling well, they attend to the treatment sessions on time and a similar percentage never thought of quitting. Conclusions: factors influencing adherence to treatment are: stable partner, family economic support, having a feeling of treatment favors recovery, feeling motivation. With respect to health team, having a positive attitude, interest in patient's attendance and giving recommendations.

Keywords: medication compliance, chemoradiotherapy, gastric neoplasms, colorectal neoplasms, radiotherapy.

1 Estudiante de Enfermería. Universidad Surcolombiana, Neiva, Colombia.

2 Enfermera, Magister en adulto anciano, Magister en educación, Profesora Facultad de Salud. Universidad Surcolombiana. Datos de contacto: Alix Yaneth Perdomo Romero , correo electrónico: alixyaneth@yahoo.es

Recibido: 18/12/2013 - Revisado: 29/05/14 - Aceptado: 30/06/2014 


\section{Introducción}

Actualmente el cáncer gástrico y colorrectal ocupan por su frecuencia a nivel mundial, la segunda y cuarta causa más frecuente de neoplasias respectivamente. En el departamento del Huila según la OMS, entre 2002 y 2006 la incidencia del cáncer colorrectal, fue de 70 por año, en hombres se mostró una incidencia de 37 casos anuales y en mujeres 33 casos $^{[1]}$. Ahora bien, en cáncer gástrico según el Instituto Nacional de Cancerología, durante el quinquenio 2007 2011 se presentaron 777 casos en hombres y mujeres. Al ser estas patologías de alta incidencia y mortalidad, el Estado y algunas organizaciones se han dado cuenta de que el cáncer se ha convertido en el problema más grande de salud en el país ${ }^{[2]}$.

Dentro de los tratamientos utilizados en los pacientes oncológicos se encuentra la cirugía, quimioterapia y/o radioterapia. Se ha demostrado que éstos últimos generan grandes niveles de incertidumbre ${ }^{[3]}$ y es el equipo de salud y la familia un apoyo constante y positivo para continuar el proceso.

Además, la capacidad de los pacientes para seguir los tratamientos de una manera óptima, se ve comprometida con frecuencia por los factores: socioeconómicos, del equipo de salud, del tratamiento, propios de la enfermedad y del paciente. Estos factores requieren un cambio de conducta en todos los niveles, tanto para el personal de salud quien debe visualizar el paciente como un ser holístico, como para el paciente, pues éste, debe estar en capacidad de incorporar el tratamiento a su estilo de vida ${ }^{[4]}$.

En el departamento no se cuenta con este tipo de estudio, por esta razón, es necesario que las instituciones de salud y los profesionales del área, conozcan los factores que influyen en la adherencia a los tratamientos y de esta manera se establezcan estrategias que permitan favorecerla.

En el presente estudio se describen los factores socioeconómicos, del equipo de salud, del tratamiento, propios de la enfermedad y del paciente, que permitan la adherencia al tratamiento de quimioterapia y radioterapia en pacientes con cáncer.

\section{Material y métodos}

Estudio descriptivo con enfoque cuantitativo de corte transversal que pretende describir los factores relacionados con la adherencia al tratamiento en pacientes diagnosticados con cáncer gástrico y colorrectal, que se encontraban en tratamiento activo de quimioterapia y radioterapia en la Unidad de Cancerología del Hospital Universitario Hernando Moncaleano Perdomo de Neiva, Centro Oncológico Liga Contra el Cáncer Seccional Huila y Unidad Oncológica Surcolombiana, en los meses de julio - octubre del 2013.

Esta población cumplía con los siguientes criterios de inclusión: Pacientes mayores de 18 años de edad, con diagnóstico de cáncer gástrico y colorrectal a partir del segundo ciclo de tratamiento activo de quimioterapia y/o el 50\% de tratamiento con radioterapia, y/o concomitancia. Se excluyeron aquellos pacientes que no desearon participar del estudio, que no habían iniciado tratamiento de quimioterapia, radioterapia y/o concomitancia y que se encontraban en su primer ciclo de tratamiento.

El instrumento elaborado por los investigadores, está compuesto por 48 ítems que evaluaron las características sociodemográficas y los factores socioeconómicos, del equipo de salud, del tratamiento y del paciente, reseñados en la literatura como influyentes en la adherencia al tratamiento. Para su validación fue enviado a juicio de expertos, quienes evaluaron criterios de pertinencia, claridad, coherencia y redacción. Posteriormente se realizó prueba piloto a 11 pacientes de la Unidad Oncológica del Hospital Universitario Hernando Moncaleano Perdomo que cumplieron con los criterios de inclusión.

La información fue organizada mediante una matriz de datos realizada en Microsoft Excel 2010, y el análisis de los datos fue procesado por el paquete estadístico SPSS versión 19.0 a través de estadística descriptiva.

\section{Consideraciones éticas}

Esta investigación fue aprobada por el Comité de ética de la Facultad de Salud y del Hospital Universitario Hernando Moncaleano Perdomo y aceptada por el Centro Oncológico Liga Contra el Cáncer Seccional Huila y Unidad Oncológica Surcolombiana.

Según la Resolución 8430 de $1993^{[5]}$, el presente estudio es considerado sin riesgo, ya que no trata aspectos sensitivos de la conducta y no realiza ninguna intervención o modificación intencionada de las variables biológicas, fisiológicas, sicológicas o sociales de los pacientes diagnosticados con cáncer gástrico participantes de esta investigación.

Está apoyada bajo la ley 911 de $2004^{[6]}$, mediante la que establece la responsabilidad del profesional de Enfermería en investigación y a partir de la cual se tienen en cuenta las disposiciones y principios éticos de dignidad, los derechos de los seres humanos, respeto por la persona, sin utilizar personas jurídicamente incapaces, totalidad e integridad, beneficencia, justicia, precaución y confidencialidad a través de la asignación de un código consecutivo que solo será conocido por los investigadores.

\section{Resultados}

La muestra consta de 35 pacientes, de los cuales el 65,7\% tenían diagnóstico de cáncer gástrico y el 34,3\% cáncer colorrectal.

La presentación de los resultados, se encuentra subdividida en características sociodemográficas y en los factores que influyen en la adherencia al tratamiento.

Las características sociodemográficas describen la población de estudio en el ámbito económico y social (tabla 1).

\section{Factores socioeconómicos que influyen en la adherencia al tratamiento}

Más del 50\% de los pacientes encuestados dependen económicamente de otra persona y ganan menos de $\$ 500.000$ al mes. Un $31,4 \%$ de la población tiene un ingreso mensual de \$500.001 $\$ 1.000 .000$ y el $11,4 \%$ entre $\$ 1.000 .001-\$ 3.000 .000$

El 66\% de la población siempre cuenta con el apoyo económico de su familia o personas allegadas para cubrir gastos derivados de su tratamiento y un $29 \%$ a veces cuenta con ese apoyo, esto se relaciona con que un $60 \%$ siempre cuenta con los medios económicos para llegar al lugar donde recibe el tratamiento y un $37 \%$ a veces.

\section{Factores del equipo de salud que influyen en la adherencia al tratamiento}

El 50\% de la población encuestada nunca ha presentado dificultades con la autorización de su tratamiento, aunque un $37 \%$ a veces la presenta; sobre todo al inicio del tratamiento.

El total de los pacientes refieren que el equipo de salud demuestra una actitud positiva cuando se les brinda el tratamiento. 
Tabla 1. Características Sociodemográficas

\begin{tabular}{|c|c|c|c|c|}
\hline Variable & Categoría & $\mathrm{n}$ & $\%$ & Total \% \\
\hline \multirow[t]{2}{*}{ SEXO } & Masculino & 19 & 54,3 & \\
\hline & Femenino & 16 & 45,7 & 100 \\
\hline \multirow[t]{5}{*}{ EDAD } & $20-30$ años & 1 & 2,9 & \\
\hline & $31-40$ años & 3 & 8,6 & \\
\hline & $41-50$ años & 5 & 14,3 & 100 \\
\hline & $51-60$ años & 11 & 31,4 & \\
\hline & Mayor de 60 años & 15 & 42,9 & \\
\hline \multirow[t]{6}{*}{ OCUPACIÓN } & Estudiante & 1 & 2,9 & \\
\hline & Desempleado & 2 & 5,7 & \\
\hline & Empleado & 4 & 11,4 & \\
\hline & Pensionado & 5 & 14,3 & 100 \\
\hline & Hogar & 11 & 31,4 & \\
\hline & Trabajador independiente & 12 & 34,3 & \\
\hline \multirow[t]{2}{*}{ ZONA DE RESIDENCIA } & Urbano & 22 & 62,9 & \\
\hline & Rural & 13 & 37,1 & 100 \\
\hline \multirow[t]{5}{*}{ ESTADO CIVIL } & Casado & 22 & 62,9 & \\
\hline & Unión libre & 6 & 17,1 & \\
\hline & Soltero & 5 & 14,3 & 100 \\
\hline & Separado & 1 & 2,9 & \\
\hline & Viudo & 1 & 2,9 & \\
\hline \multirow[t]{6}{*}{ ESCOLARIDAD } & Primaria completa & 10 & 28,6 & \\
\hline & Primaria incompleta & 11 & 31,4 & \\
\hline & Bachillerato completo & 6 & 17,1 & \\
\hline & Bachillerato incompleto & 6 & 17,1 & 100 \\
\hline & Técnico completo & 1 & 2,9 & \\
\hline & Técnico incompleto & 1 & 2,9 & \\
\hline \multirow[t]{4}{*}{ TIPO DE SEGURIDAD } & Subsidiado & 19 & 54,3 & \\
\hline & Contributivo & 12 & 34,3 & 100 \\
\hline & Régimen especial & 3 & 8,6 & \\
\hline & Medicina prepagada & 1 & 2,9 & \\
\hline
\end{tabular}

E1 94\% de los participantes considera que el equipo de salud muestra interés por su asistencia al tratamiento, lo que a su vez se relaciona con el $69 \%$ que siempre ha recibido llamadas para que asista. Por otro lado un $26 \%$ a veces las recibe, relacionándose con la puntualidad de los pacientes para asistir al tratamiento.

Más del $80 \%$ expresan que siempre el enfermero (a) emplea suficiente tiempo para dar recomendaciones claras sobre el tratamiento, además resuelve las preguntas e inquietudes derivadas de este.

\section{Factores del tratamiento que influyen en la adherencia al tratamiento}

El 91\% de los pacientes manifiestan que el tratamiento siempre ha ayudado a su recuperación.

E1 86\% enuncia que siempre asiste a su tratamiento aun cuando se sienten mal. Esto a su vez concuerda con el $86 \%$ de los pacientes que nunca han pensado en abandonar el tratamiento, siendo esto un factor positivo para la adherencia al tratamiento. 
Tabla 2.Factores propios de la enfermedad que influyen en la adherencia al tratamiento

\begin{tabular}{lccc} 
Variable & Categoría & Número & Porcentaje \\
Diagnóstico & Gástrico & 23 & 65,7 \\
& Colorrectal & 12 & 34,3 \\
\hline Estadio de la enfermedad & I & 2 & 5,7 \\
& III & 6 & 17,1 \\
& IV & 17 & 48,6 \\
& Si & 10 & 28,6 \\
\hline ¿Conoce sobre su enfermedad? & No & 30 & 85,7 \\
& Curativo & 5 & 94,3 \\
\hline ¿Conoce el objetivo del tratamiento? & Paliativo & 33 & 5,7 \\
\hline ¿Esto ha influido para continuar el tratamiento? & Si & 2 & 94,3 \\
& No & 33 & 5,7 \\
\hline
\end{tabular}

El personal de salud siempre brinda solución a las molestias ocasionadas por el tratamiento referido por un $74 \%$ de los participantes.

Las reacciones adversas más frecuentes que referencian los 35 participantes fueron: $63 \%$ pérdida del apetito y náuseas y vómito, seguida por dolor, diarrea y fatiga con un $51,4 \%$ cada una, siendo las de menor proporción resequedad de la piel y estreñimiento con un $40 \%$ y $28,5 \%$ respectivamente (Tabla 2 ).

Es de resaltar que el 77,2\% de los pacientes que se encontraban en el estadio III y IV aun cuando la finalidad del tratamiento era paliativo, solamente el $5.8 \%$ de ellos lo percibían de esta manera.

Factores propios del paciente que influyen en la adherencia al tratamiento

El 91,4\% de los pacientes asiste puntualmente a las sesiones de tratamiento.

Al $60 \%$ de los pacientes el tratamiento lo limita para realizar sus actividades diarias, el $28,6 \%$ refiere que el tratamiento lo limita algunas veces.

El $57,1 \%$ de los pacientes refirieron que su estilo de vida ha cambiado debido al tratamiento, seguido del $25,7 \%$ que considera algunas veces. Sin embargo un $17,1 \%(n=6)$ nunca ha cambiado su estilo de vida, resaltando que el $83,3 \%(n=5)$ del anterior porcentaje se encuentran en estadios avanzados.

Un $94,3 \%$ de la población se siente motivada a cumplir con su tratamiento. La orientación clara, oportuna y la confianza que brinda el personal de salud a la hora de realizar los procedimientos hace que un $68 \%$ de los pacientes encuestados refieran nunca sentir temor cuando asisten al tratamiento y que a su vez se relaciona con el $91,4 \%$ que se siente tranquilo cuando recibe el tratamiento. E1 28,6\% manifestó algunas veces sentir temor, esto relacionado con la etapa inicial del tratamiento.

El 54,3\% de los encuestados manifiestan nunca sentir tristeza cuando reciben el tratamiento. Aunque existe un 34,3\% que refirió algunas veces y un $11,4 \%$ que siempre la experimenta.
Las respuestas de los pacientes acerca de si el tratamiento ha ocasionado cambios en el aspecto físico están divididas en porcentajes iguales, puesto que un $37,1 \%$ de los encuestados consideran que siempre lo ha hecho y un porcentaje igual nunca.

El 82,9\% de los participantes siempre cuentan con el acompañamiento de algún familiar, por lo general esposo (a), hijo (a), nuera o yerno. Lo cual se relaciona con el $80 \%$ que refirió nunca contar con la compañía de un amigo, demostrando que es la familia quien asume esta responsabilidad. El 14,3\% algunas veces tiene compañía de algún familiar.

El 88,6\% siempre considera que el estar acompañado favorece la asistencia al tratamiento, lo cual se relaciona con el 94,3\% que refiere recibir siempre motivación por parte de su familia para continuar con el mismo.

\section{Discusión}

La adherencia terapéutica es una de las estrategias más representativas para el tratamiento de las enfermedades crónicas, entre estas el cáncer; puesto que permite la adaptación y continuidad de los pacientes a la recuperación y mejoramiento de su calidad de vida. Además, es un fenómeno complejo que está condicionado por múltiples factores de diversa naturaleza ${ }^{[7]}$, los cuales se describen en la presente investigación como características sociodemográficas, factores socioeconómicos, del equipo de salud, del tratamiento, propios del paciente y de la enfermedad.

Los resultados encontrados con respecto a la edad corresponden con otros autores, ${ }^{[8,9]}$ quienes afirman que los pacientes en edades avanzadas presentan mejores tasas de adherencia que los de edades intermedias, evidenciado en el presente estudio donde la mayoría de los encuestados $(74,3 \%)$ eran mayores de 51 años.

En cuanto al estado civil, el tener una pareja estable permite la asistencia y continuidad a los tratamientos, lo que se puede contrastar con otro estudio, ${ }^{[10]}$ el cual afirma que el estado civil casado 
es una práctica de apoyo que tiene un efecto significativo para la adherencia al tratamiento.

La familia como principal fuente de apoyo económico y afectivo constituye la primera red de apoyo social y juega un papel fundamental en el fortalecimiento de la adherencia, porque motiva al paciente a ver el tratamiento como una forma de vida, afirmado por los autores ${ }^{[11,12]}$; relacionado con el presente estudio, donde el $66 \%$, siempre cuenta con el apoyo económico de su familia para cubrir gastos derivados de su tratamiento, además, ellos, quienes brindan el acompañamiento y por tanto asumen esta responsabilidad afectiva y material.

El presente estudio encontró que el $54 \%$ de los pacientes nunca presentó dificultades con la autorización de su tratamiento, siendo una fortaleza para la adherencia al tratamiento, sin embargo, quienes enunciaron dificultades, refirieron que éstas se produjeron al inicio del mismo, Suarez menciona en su estudio que los pacientes abandonan su tratamiento por causas relacionadas con el trámite de autorizaciones, lo cual es una condición particular del sistema de seguridad social en Colombia de cómo se ofertan los servicios de salud para el tratamiento del cáncer ${ }^{[13]}$.

Algunos autores, ${ }^{[14-16]}$ afirman que aspectos como el trato deshumanizado, malas actitudes e indisposición, limitan la adherencia al tratamiento, además, destacan la comunicación eficaz que debe existir entre el profesional de salud y el paciente, en donde el hecho de proporcionar la información necesaria, clara y oportuna favorece el comportamiento de la adherencia; aspectos importantes que se señalan en los resultados de la presente investigación, pues el equipo de salud demostró una actitud positiva cuando le brindó el tratamiento, interés para lograr la asistencia de los pacientes al mismo y el personal médico y de enfermería dio recomendaciones claras, oportunas, respondiendo a las inquietudes derivadas del tratamiento.

Se observó en la investigación, que más del 90\% de los pacientes consideraron que el tratamiento siempre ha ayudado a su recuperación, además, un 94,3\% lo perciben como curativo, favoreciendo así la adherencia; esto se relaciona con lo que menciona el estudio $^{[16]}$, donde afirma que el paciente oncológico al percibir como efectivo el tratamiento al cual está siendo sometido, le permite evaluarlo como positivo pues controla el progreso de la enfermedad y evita así la deserción terapéutica.

Según lo descrito por los autores ${ }^{[16,17]}$, los efectos colaterales de los medicamentos pueden actuar como estímulos aversivos y producir así un descenso en la adherencia, aun cuando el paciente se empieza a encontrar bien. Para el presente estudio a pesar de que el $86 \%$ de la población encuestada se sentía mal, asistía puntualmente a las sesiones del tratamiento y por tanto un porcentaje similar nunca ha pensado en abandonarlo, esto resulta ser un factor positivo para la adherencia al tratamiento.

En el artículo cumplimiento e incumplimiento del tratamiento antihipertensivo desde la perspectiva del paciente ${ }^{[18]}$, se corrobora la importancia de los conocimientos del paciente acerca de la enfermedad como un aspecto favorecedor del cumplimiento, esto comparado con la presente investigación evidencia que el $85,7 \%$ de los pacientes que tuvieron conocimientos generales de la enfermedad se vean influenciados positivamente en la asistencia al tratamiento.

De acuerdo con los autores ${ }^{[10,19]}$, los comportamientos de adherencia hacen referencia a la incorporación o mantenimiento de hábitos en el estilo de vida, la modificación de aquellos que puedan constituir un factor de riesgo para la progresión de la enfermedad y que pueden poner en peligro su salud. Al encontrar en este estudio que un $82,8 \%$ de los pacientes han cambiado sus estilos de vida debido al tratamiento, se convierte en un comportamiento favorable para la adherencia de acuerdo con los autores.

Los autore ${ }^{[7]}$ destacan como aspectos motivacionales de los pacientes, deseos de vivir más, sentirse bien con el objetivo de mejorar la calidad de vida, siendo éstas necesidades de un valor significativo para la persona; sumado a esto, los autores aprecian que los pacientes perciben el temor como un factor que interfiere en el cumplimiento terapéutico, debido a los efectos secundarios que el tratamiento produce. En relación con la presente investigación, un $94,3 \%$ de la población encuestada se siente motivada a cumplir con el tratamiento, es decir tienen grandes expectativas por su recuperación; agregado a esto se evidencia que un $68 \%$ de los pacientes refieren nunca haber sentido temor durante el tratamiento, por lo que se puede deducir que los efectos secundarios no representan un factor que desencadene temor en ellos.

\section{Conclusión}

El tener edad avanzada y el contar con una pareja estable (casado o unión libre), son las características sociodemográficas de mayor influencia en la adherencia a los tratamientos del cáncer gástrico y colorrectal.

La familia y el equipo de salud como grandes influyentes en el fortalecimiento de la adherencia, aseguran al paciente dar continuidad al tratamiento, utilizando como medios la motivación y el apoyo tanto afectivo como económico. Por tanto los factores del equipo de salud, socioeconómico y propio del paciente cobran también gran significancia en el cumplimiento del tratamiento.

Es importante que enfermería dentro del plan de cuidados fortalezca las estrategias de comunicación asertiva que deben existir entre el equipo de salud, el paciente y su familia, con el fin de mejorar el proceso de adherencia en pro de la calidad de vida del paciente.

\section{Agradecimientos}

A las Unidades Oncológicas: Hospital Universitario Hernando Moncaleano Perdomo de Neiva, Unidad Oncológica Surcolombiana y el Centro Oncológico de la Liga contra el Cáncer seccional Huila, quienes nos permitieron acceder a la población de estudio, herramienta necesaria e imprescindible para la elaboración de esta investigación.

A las enfermeras oncólogas Ruth Díaz, Liliana Verú, Maricela Polanía y Nohemí Cuellar, quienes de manera desinteresada y generosa, fueron la fuente constante de comunicación para lograr el vínculo entre los pacientes e investigadores. Así como a nuestros pacientes que de forma voluntaria y contribuyente compartieron su tiempo y vivencias personales para el enriquecimiento de los resultados de la investigación.

A los profesionales expertos en investigación, quienes dedicaron parte de su tiempo para validar el instrumento con el cual se recolectó la información, junto con aquellos que apoyaron la parte estadística del estudio.

\section{Bibliografia}

1. Rada Escobar, C.V., Incidencia estimada y mortalidad por cáncer en Colombia, 2002-2006. 2009, Instituto Nacional de Cancerologia: Bogota. p. 98. 
2. Ospina Ospina, I.C., Panorama General del Cancer en Colombia. Altos en Linea, 2013. 4(8).

3. Ehrenzweig Sánchez, Y., Modelos de cognición social y adherencia terapéutica en pacientes con cáncer. Avances en Psicología Latinoamericana, 2007. 25:07-21.

4. Soria Trujano, R., et al., Estrés familiar y adherencia terapéutica en pacientes con enfermedades crónicas. Alternativas en Psicologia, 2012(26):7.

5. RESOLUCION № 8430 DE 1993, in 8430, M.d.S.R.d. Colombia, Editor. 1993, Ministerio de Salud: Bogotá.

6. Ley 911 de 2004, C.d. Colombia, Editor. 2004, Ministerio de Proteccion Social: Bogota.

7. Martin Alfonso, L.G.A., J.A, La investigación de la adherencia terapéutica como un problema de la psicología de la salud. Psicologia y Salud, 2004. 14(1).

8. Caceres, M.M., Factores de riesgo para abandono (no adherencia) del tratamiento antituberculoso. Revista Med UNAB, 2004. 7:4.

9. Garcia Leguizamon, M., Factores relacionados con la adherencia al tratamiento farmacológico y no farmacológico en pacientes con post infarto agudo de miocardio en una Institución de cuarto nivel, Bogotá durante el mes de Agosto de 2008, in Enfermeria Clinica. 2008, Pontifica Universidad Javeriana: Bogotá.

10. DiMatteo, M.R., Social support and patient adherence to medical treatment: a meta-analysis. Health Psychol, 2004. 23(2):207-18.

11. Castaño-Castrillón, J.J., et al., Adherencia al tratamiento de pacientes hipertensos atendidos en Assbasalud ESE, Manizales (Colombia) 2011. Revista de la Facultad de Medicina, 2012. 60:179-197.
12. Saad Acosta, C., et al., Adherencia de los pacientes con insuficiencia renal crónica a las sesiones de hemodiálisis del Hospital Militar Central, Bogotá, DC, 2006 2007. Rev. colomb. enferm, 2008. 3(3):61-67.

13. Suárez, A., et al., Abandono del tratamiento: una causa de muerte evitable en el niño con cáncer. Revista Colombiana de Cancerología, 2011. 15(1):22-29.

14. Muñoz Sánchez, A.I., Ó.A. Cruz Martínez, and Y.L. Rubiano Mesa, Trabajadores de la salud y sus significados en torno a la adherencia al tratamiento de la tuberculosis. Enfermería Global, 2013. 12(3): p. 86-108.

15. Beltran Cortes, A. and M. Leal Quintero, Factores relacionados con la adherencia al tratamiento farmacológico en pacientes con diagnóstico de depresión en una institución de salud de Bogotá., in Departamento de Clinica. 2009, Pontificia Universidad Javeriana: Bogota.

16. Urzúa M., A., A. Marmolejo C., and C. Barr D., Validación de una escala para evaluar factores vinculados a la adherencia terapéutica en pacientes oncológicos. 2011. Vol. 11.

17. Ortego Maté, M.C., La adherencia al tratamiento. Variables implicadas. Educare, 2004. 8.

18. Martín Alfonso, L., et al., Cumplimiento e incumplimiento del tratamiento antihipertensivo desde la perspectiva del paciente. Revista Cubana de Salud Pública, 2007. 33: p. 0-0.

19. Varela Arévalo, M., El reto de evaluar la adherencia al tratamiento en la hipertensión arterial. Pensamiento Psicológico, 2010. 7(14):127-40. 